\title{
Systematic review of pre-clinical chronic myeloid leukaemia
}

\author{
Jew Win Kuan ${ }^{1}\left[\right.$ (D) Anselm Ting Su² Chooi Fun Leong $^{3} \cdot$ Motomi Osato $^{4} \cdot$ Goro Sashida $^{4}$ \\ Received: 13 June 2018 / Revised: 31 August 2018 / Accepted: 3 September 2018 / Published online: 14 September 2018 \\ (c) The Japanese Society of Hematology 2018
}

\begin{abstract}
Background Studies of a provisional entity pre-clinical chronic myeloid leukaemia (CML), which precedes chronic phase (CP) without leucocytosis or blood/marrow feature of CML CP, has been increasing.

Objective To perform a systematic review of pre-clinical CML and analysis the data relevant to disease progression to CML CP.

Method We performed a literature search on 16 July 2017 using EBSCOhost Research Databases interface and Western Pacific Region Index Medicus. Two authors selected the studies, extracted the data and evaluated the quality of studies using an 8-item tool, independently. The outcomes were percentage of Philadelphia chromosome in the number of metaphases examined ( $\mathrm{Ph} \%)$, correlation between $\mathrm{Ph} \%$ and blood count and time progress to CML.

Result Our initial search returned 4770 studies. A total of 10 studies with a total 17 subjects were included. The lowest $\mathrm{Ph} \%$, which eventually progresses to CML, was $10 \%$. Absolute basophil count seemed to correlate better with $\mathrm{Ph} \%$ compared to total white cell and absolute eosinophil count. The time from the first documented pre-clinical CML to CML ranged from 12 to 48 months. The overall quality of the included studies was average.
\end{abstract}

Conclusion This is the first systematic review on pre-clinical CML. This entity requires additional large-scale studies.

Keywords Chronic myeloid leukaemia $\cdot$ Pre-clinical $\cdot$ Asymptomatic $\cdot$ Smoldering

\section{Introduction}

Chronic myeloid leukaemia (CML) is staged into chronic phase (CP), accelerated phase (AP) and blast crisis (BC). According to World Health Organization (WHO) classification 2017, CML, BCR-ABL1-positive CP is diagnosed when there is Philadelphia $(\mathrm{Ph})$ chromosome or $B C R-A B L 1$ (as the result of translocation between the $v$-abl Abelson murine leukemia viral oncogene homolog 1 (ABL1) gene

Jew Win Kuan

kuanjewwin@gmail.com

1 Department of Medicine, Faculty of Medicine and Health Sciences, Universiti Malaysia Sarawak, 94300 Kota Samarahan, Sarawak, Malaysia

2 Department of Community Medicine and Public Health, Faculty of Medicine and Health Sciences, Universiti Malaysia Sarawak, Kota Samarahan, Sarawak, Malaysia

3 Department of Pathology, Universiti Kebangsaan Malaysia Medical Center, Kuala Lumpur, Malaysia

4 International Research Center for Medical Sciences, Kumamoto University, Kumamoto, Japan on chromosome 9 and the breakpoint cluster region $(B C R)$ gene on chromosome 22) presence together with other features, i.e. leukocytosis (total white cell count (TWC) of $12-1000 \times 10^{9} / \mathrm{L}$ ), different stages of neutrophil maturation (peaks in myelocytes and segmented neutrophils) in peripheral blood film (PBF) and hypercellularity and granulocytic proliferation with maturation similar to PBF in bone marrow aspiration (BMA) [3]. Absolute basophilia and eosinophilia are common [3]. Blast count in peripheral blood (PB) or BMA is less than $10 \%$ [3]. Splenomegaly and symptoms are usually present when total white cell count (TWC) is more than $50 \times 10^{9} / \mathrm{L}[3,4]$. About $20-50 \%$ of the CML patients were asymptomatic at diagnosis when leukocytosis was detected incidentally, frequently during health check $[3,5]$.

Since the discovery of $\mathrm{Ph}$ chromosome by cytogenetic study in 1960 [6], there are more and more studies reported on a provisional entity of CML, a condition before CP, without leucocytosis or PBF or BMA feature of CML CP. Various nomenclature has been used, such as "pre-clinical", "subclinical", "pre-leukaemic" or "smoldering" CML. For the purpose of this review, we refer this entity as "pre-clinical". Pre-clinical CML was usually "diagnosed" when $\mathrm{Ph}$ 